\title{
Influencia en el alumnado del Trabajo Fin de Grado con datos originales en el Grado en Fisioterapia: estudio cualitativo Influence on the students of the Degree Thesis with original data in the Physical Therapy Degree: qualitative study
}

\author{
Daiana Priscila Rodrigues-de-Souza ${ }^{1}$, Francisco Alburquerque-Sendín ${ }^{1,2}$ \\ drodrigues@uco.es, falburquerque@uco.es \\ ${ }^{1}$ Departamento de Enfermería, Farmacología y \\ Fisioterapia \\ Universidad de Córdoba \\ Córdoba, España \\ ${ }^{2}$ GC05 Enfermedades autoinmunes sistémicas- \\ inflamatorias crónicas del aparato locomotor y \\ tejido conectivo \\ IMIBIC \\ Córdoba, España
}

Resumen- La posibilidad de realizar Trabajo de Fin de Grado (TFG) con datos originales de investigación, o con otras metodologías y modalidades, podría tener influencia en la perspectiva del alumno respecto a su visión del Grado de Fisioterapia y su profesión. Se ha diseñado un estudio cualitativo para identificar posibles diferencias en dicha perspectiva en el Grado en Fisioterapia de la Universidad de Córdoba. Los alumnos que han manejado TFG con datos originales manifiestan una gran satisfacción con su TFG y aseguran que les ha modificado su perspectiva, al haber conocido, personalmente, cómo se genera el conocimiento académico y clínico de la Fisioterapia. Los que no han manejado datos originales también se muestran igualmente satisfechos, pero no reconocen cambios en forma de percibir la profesión ni en ámbito académico. Así, la realización de TFGs con datos originales puede tener repercusiones beneficiosas en la perspectiva académica y profesional del alumno en el Grado en Fisioterapia.

\section{Palabras clave: Trabajo Fin de Grado, Competencias investigadoras, Entorno profesional en Fisioterapia}

Abstract- The possibility of carrying out a Degree Thesis (DT) with original research data, or with other methodologies and modalities, could have an influence on the student's perspective regarding their vision of the Degree in Physiotherapy and their profession. A qualitative study has been designed to identify possible differences in this perspective in the Degree in Physiotherapy at the University of Cordoba. The students who have worked with DT based on original data express great satisfaction with their DT and state that it has modified their perspective, because they have known how academic and clinical knowledge of Physiotherapy is generated. Those who have not handled original data are also equally satisfied, but do not recognize changes in the way they perceive the profession or in the academic environment. Thus, the realization of DT with original data can have beneficial repercussions in the academic and professional perspective of the student in the Degree in Physiotherapy.

Keywords: Degree Thesis, Research Competences, Professional Environment in Physical Therapy

\section{INTRODUCCIÓN}

Durante el $4^{\circ}$ curso del Grado en Fisioterapia se desarrollan asignaturas, contenidos y competencias de carácter diferencial al de los cursos anteriores, entre las que se encuentran las destrezas clínicas, adquiridas en los Practicum, o competencias transversales e integradoras, como las que ampara el Trabajo de Fin de Grado (TFG). Este escenario requiere de estrategias metodológicas y evaluadoras más ambiciosas e innovadoras, como pueden las Examen de Competencias Objetivo y Estructurado (ECOE), destinadas la evaluación de las competencias clínicas, de reciente instauración en algunos Grados de Fisioterapia españoles (Rodrigues de Souza et al., 2019). En lo referente a los TFG, los requerimientos descritos en el Real Decreto 1393/2007, de 29 de octubre, por el que se establece la ordenación de las enseñanzas universitarias oficiales, y su modificación Real Decreto 861/2010, de 2 de julio, determinan que el TFG tiene un carácter obligatorio, ha de constar de entre 6 y 30 ECTS, se ha de realizar al final del Plan de estudios. Además, específicamente en el Grado en Fisioterapia, según la ORDEN CIN/2135/2008, de 3 de julio, por la que se establecen los requisitos para la verificación de los títulos universitarios oficiales que habiliten para el ejercicio de la profesión de Fisioterapeuta, el TFG, que se encuentra en el módulo Prácticas Tuteladas y Trabajo de Fin de Grado, permitirá adquirir la Competencia de "Presentación y defensa ante el Tribunal universitario de un proyecto de fin de grado, consistente en un ejercicio de integración de los contenidos formativos recibidos y las competencias adquiridas".

Además, en el Grado de Fisioterapia de la Universidad de Córdoba (UCO), la Guía académica de la asignatura TFG (Facultad de Medicina y Enfermería, 2020) recoge las siguientes competencias, que identifican tanto el ámbito profesional, como el investigador:

- CB04: Que los estudiantes puedan transmitir información, ideas, problemas y soluciones a un público tanto especializado como no especializado.

- CG15: Presentación y defensa ante el Tribunal universitario de un proyecto de fin de grado, consistente en un 
ejercicio de integración de los contenidos formativos recibidos $\mathrm{y}$ las competencias adquiridas fomentando actividades profesionales que dinamicen la investigación en fisioterapia.

- CG17: Comprender la importancia de actualizar los conocimientos, habilidades, destrezas y actitudes que integran las competencias profesionales del fisioterapeuta.

- CG19: Comunicarse de modo efectivo y claro, tanto de forma oral como escrita, con los usuarios del sistema

- CT1: Acreditar el uso y dominio de una lengua extranjera

- CE20: Presentación y defensa ante el Tribunal universitario de un proyecto de fin de grado, consistente en un ejercicio de integración de los contenidos formativos recibidos y las competencias adquiridas

Respecto a los temas, la propia Guía determina que deberán "abordar el futuro profesional, así mismo deben incorporar las inquietudes de los estudiantes y su identificación con el trabajo profesional, siendo su enfoque genérico el desarrollo de planes de cuidados, estudio de casos y análisis crítico y científico en fisioterapia", lo que supone un proceso único en la Titulación.

Respecto a los formatos del TFG del Grado en Fisioterapia, se permite una alta diversidad de formatos, con enfoques profesionales, bibliográficos, investigadores, e incluso libres (Arbós Berenguer, 2020). De todos ellos, en la UCO se permiten los siguientes:

1. Formato de Presentación de Caso Clínico.

2. Formato de descripción de un Protocolo de tratamiento o prevención.

3. Formato de Emprendimiento.

4. Formato de Proyecto de Investigación.

5. Formato de Trabajo de Investigación.

6. Formato de Revisión Bibliográfica.

7. Formato para los casos de trabajos publicados.

8. Formato para los casos de comunicaciones a Congresos.

9. Otros formatos que no se ajusten a los anteriores.

Como puede observarse, los Formatos 1, 5, 7, y 8, además de, en determinados casos el Formato 9, precisan de la adquisición y manejo de datos primarios obtenidos de pacientes (TFGprimarios), mientras el resto no requeriría de tales datos (TFGnoPrimarios). No obstante, no se ha determinado en qué nivel o de qué modo contribuye cada uno de los Formatos al desarrollo de las competencias atribuidas al TFG.

Todo ello, unido a la pandemia por la COVID-19, ha supuesto un esfuerzo reflexivo y adaptativo, que ha afectado a los métodos y evaluaciones de los Grados (De Miguel Rubio et al., 2019), quizá con mayor intensidad en el $4^{\circ}$ curso, dada la especificidad y originalidad de sus materias, como ha ocurrido con las ECOE presenciales, que han pasado a ser virtuales (Alburquerque-Sendín et al., 2020). Así, los TFG que manejaban tradicionalmente datos de investigaciones originales o primarias, se han visto abocados a construirse bajo otras modalidades, haciéndose necesaria una evaluación del rendimiento competencial que supone la realización de un modelo de TFG con manejo de datos originales, respecto a otros que no trabajen con este material, todo ello en un Grado, como el de Fisioterapia, regulado y profesionalizante, que no obliga a asumir competencias investigadoras.

\section{CONTEXTO}

Debido a lo heterogéneo de las metodologías y modalidades de realización de TFGs en el Grado en Fisioterapia en España, y particularmente en la UCO, que cuenta con 45 alumnos como máximo por promoción, es conveniente identificar qué ventajes e inconvenientes puede tener cada una de las aproximaciones. De esta forma, los alumnos matriculados en $3^{\circ}$ curso en la asignatura Proyecto de TFG, y los que en $4^{\circ}$ desarrollan la asignatura de TFG, con 6 ECTS y carácter anual, contarán con mayor información respecto a qué supone hacer unas $\mathrm{u}$ otras modalidades de TFG. Además, los profesores-tutores de TFG también podrán valorar la conveniencia de proponer unos $\mathrm{u}$ otros temas o líneas de TFGs en función de diferentes factores, como son los conocimientos propios, las capacidades del alumno, sus necesidades y disponibilidad temporal.

Para conseguir alcanzar este conocimiento, se propone identificar el nivel de satisfacción del alumnado que ha realizado TFGprimario y del que no ha realizar su TFG con otras modalidades o metodologías. Asimismo, se pretender recoger la opinión de profesores-tutores que han tutorizado, en los cursos recientes, TFGprimario y TFGnoPrimario.

\section{DESCRIPCIÓN}

Para la consecución del objetivo propuesto se ha diseñado un estudio observacional transversal, de carácter cualitativo fenomenológico (trabajo de campo con entrevista semiestructurada y análisis de contenido) que ya se ha mostrado previamente útil en la investigación en Fisioterapia (Rodriguesde-Souza et al., 2016). La fenomenología intenta comprender cómo los individuos construyen su visión del mundo y mira a través de una ventana a las experiencias de otras personas (Carpenter y Suto, 2008).

\section{A. Participantes}

Se empleó reclutamiento no probabilístico de casos consecutivos, de carácter intencional y por bola de nieve. Así se incluyeron a 6 alumnos del Grado de Fisioterapia de la UCO que cursaron TFGprimario y 6 que cursaron su TFGnoPrimario, además de 2 profesores-tutores que habían dirigido ambos tipos de TFG, todo ello en los últimos 3 cursos académicos.

\section{B. Recogida de datos}

Se realizaron entrevistas semi-estructuradas a todos los participantes, que se registraron para su posterior análisis, además de anotar aquellos otros aspectos comportamentales que se consideró oportuno. Las preguntas directas versaron sobre los siguientes aspectos:

\section{¿Cuál es tu experiencia con el TFG que has realizado?}

¿Crees que haber
TFGprimario/TFGnoPrimario te da algún valor añadido
respecto a otras modalidades?

¿Cómo puede haber influido el TFG que has realizado tu visión sobre aspectos como tu futuro profesional o el ámbito académico? 
En todo caso, se permitió la respuesta abierta y se facilitó la escucha, siendo más importantes las respuestas que las preguntas.

\section{Análisis de datos}

Se realizó una transcripción íntegra de las entrevistas. Por separado entre investigadores, se aplicó un análisis temático, identificando y clasificando el material en unidades de significado, y agrupando posteriormente éstas temas. Asimismo, se buscó el consenso entre investigadores (Murray et al., 2009; Rodrigues-de-Souza et al., 2016).

\section{Resultados}

Se identificaron 3 temas principales: Dependencia de la satisfacción con el TFG en función de su modalidad; Profundización en la adquisición de competencias en función de la modalidad del TFG; El manejo de datos primarios como aproximación del alumno a la cotidianidad de la profesión.

\section{A. Dependencia de la satisfacción con el TFG en función de su modalidad}

Todos los alumnos entrevistados manifestaron un alto grado de satisfacción con el TFG realizado, independientemente de su modalidad, así como con la tutorización de la que habían sido objeto. Incluso, en dos ocasiones, habiendo realizado un alumno un TFGprimario y otro un TFGnoPrimario se registró la frase: Repetiría tema, tutor y todo lo que he hecho en el TFG. Este grado de satisfacción no se tradujo íntegramente en la calificación obtenida en la asignatura TFG, a pesar de que ningún alumno suspendió ninguna de las convocatorias de exposición y defensa de TFG a que se presentaron, y 9 de los alumnos entrevistados no estaban satisfechos con la calificación numérica por considerarla baja, siendo 4 alumnos de TFGprimario y 5 más de TFGnoPrimario.

Además, los profesores-tutores también declararon un alto grado de satisfacción con la tutorización realizada y los resultados académicos obtenidos en ambas modalidades de TFG.

\section{B. Profundización en la adquisición de competencias en función de la modalidad del TFG}

Los alumnos de TFGnoPrimario no detectaron un importante valor añadido en la adquisición de competencias por la realización del TFG, comparado con otras asignaturas. Es más, 3 de los 6 alumnos repreguntaron y no entendían el por qué de la cuestión planteada, pensando que no habían hecho bien el TFG si no habían sentido ese valor añadido, por lo que se mostraron preocupados.

Por el contrario, los alumnos que realizaron el TFGprimario sí modificaron su forma de concebir la formación recibida hasta la fecha. Varios de ellos manifestaron un sentir homogéneo, en el que decían que ahora no sólo "sabían más", si no que también "sabían mejor". Llamó la atención que uno de los alumnos expresó: Hasta que hice el TFG pensé que me había equivocado de carrera y de profesión, pero ahora sé que no es asi.

Este cambio de perspectiva no es inusual en este ámbito concreto $\mathrm{y}$, de hecho, ya ha sido referenciado por alumnos que han realizado TFG de investigación en cualquiera de sus modalidades, ya que se sienten parte activa de cómo se genera lo que estudian y se les enseña, en un ámbito concreto de la Fisioterapia (Rodrigues de Souza y Alburquerque-Sendín, 2021).

\section{El manejo de datos primarios como aproximación del alumno a la cotidianidad de la profesión}

Al igual que en la profundización de competencias, los alumnos que realizaron TFGnoPrimario no encontraron llamativo el TFG como motor del vínculo entre lo que le pasa al paciente $\mathrm{y}$ lo que ellos estudiaban o practicaban. Consideraban el TFG como una asignatura más, también en este sentido. No obstante, tres de los alumnos que habían realizado TFGprimario indicaron que ahora conocen el recorrido desde lo que le pasa al paciente hasta que se convierte en conocimiento científico, lo que les reportaba una visión diferente de la profesión. Esta visión global también fue informada por los profesores-tutores, siendo argumentada en el sentido de que los alumnos que han realizado TFGprimario ven el cierre completo del círculo que supone la pregunta clínica hasta que se resuelve con conocimiento científico. Finalmente, dos alumnos de TFGprimario manifestaron que ahora ven la carrera académica como una opción de futuro, aunque a largo plazo.

\section{CONCLUSIONES}

Tanto los TFG con datos primarios como los que no los manejaron generan un alto grado de satisfacción por parte del alumno. La realización de TFG con datos primarios es percibida como un aprendizaje más profundo en la adquisición de competencias, lo que se considera beneficioso. Los TFG con datos primarios generan una mayor proximidad del alumno al ámbito académico, aumentando su interés por él. Los profesores-tutores de TFG de diferentes modalidades constatan que los alumnos que realizan TFG con datos primarios adquieren una visión global y más próxima a la realidad de la Fisioterapia actual.

\section{AgRADECIMIENTOS}

A nuestros alumnos, que nos enseñan lo costoso que es formarnos.

\section{REFERENCIAS}

Alburquerque-Sendín, F., Rodrigues De Souza, D., Álvarez Rivas, J., Jiménez Esquinas, R., Sánchez De La Vaquera, M. T., León Bravo, G., Sánchez Núñez, M. R., Girela López, E., Labella Quesada, F., y Jiménez Reina, L. (Noviembre de 2020). Adaptación de una ECOE presencial a modalidad on-line en el Grado en Fisioterapia debido a la COVID-19. En II Congreso Internacional de Innovación Docente e Investigación en Educación Superior, Madrid (España).

Arbós Berenguer, M. T. (2020). ¿Cómo sobrevivir al TFG en el Grado de Fisioterapia? En J. Molina Mula, A. Amézcua Sánchez (Ed.). Sobrevivir al TFG en Fisioterapia (pp. 27-52). Madrid (España). Ed. FUDEN.

Carpenter, C., y Suto, M. (2008). Qualitative research for occupational and physical therapists: A practical guide. Oxford, Reino Unido: Black-Well Publishing.

De Miguel Rubio, A., Rodrigues De Souza, D., Álvarez Rivas, J., y Alburquerque-Sendín F. (Noviembre de 2020). Pandemia y Universidad: experiencia del profesorado del 
Grado de Fisioterapia de la Universidad de Córdoba, estudio cualitativo. En II Congreso Internacional de Innovación Docente e Investigación en Educación Superior, Madrid (España).

Facultad de Medicina y Enfermería (2020). Gruía Académica de la asignatura Trabajo Fin de Grado, Grado de Fisioterapia, Universidad de Córdoba. Recuperado de https://www.uco.es/organiza/centros/medicinayenfermeri a/images/documentos/fisioterapia/guias_docentes/GUIA _DOCENTE_TFG_Fisioterapia_20_21.pdf

Murray, S. A., Kendall, M., Carduff, E., Worth, A., Harris, F. M, Lloyd, A., ... Sheikh, A. (2009). Use of serial qualitative interviews to understand patients' evolving experiences and needs. BMJ.; 339, b3702.

Rodrigues de Souza, D. P., Jiménez Reina, L., Labella Quesada, F., Girela López, E., Chacón Invernó, J. y Alburquerque Sendín, F.. (Mayo de 2019). Integrative development of an osce in the degree in physiotherapy: preamble, design and presentation. En I Congreso Internacional de Investigación en Tutelaje Clínico, Málaga (España).

Rodrigues de Souza, D., y Alburquerque-Sendín F. (Abril de 2021). Experiencia Discente En La Elaboración De Trabajos De Fin De Grado De Tipo Investigador En El Grado En Fisioterapia. En I Congreso Internacional De Calidad E Innovación Docente En Ciencias De La Salud, Murcia (España).

Rodrigues-de-Souza, D. P, Palacios-Ceña, D., MoroGutiérrez, L., Camargo, P. R., Salvini, T. F., y Alburquerque-Sendín, F. (2016). Socio-cultural factors and experience of Chronic Low Back Pain: a Spanish and Brazilian Patients' Perspective. A Qualitative Study. PLOS one. 11(7), e0159554. 